\title{
Bifunctional Methylenetetrahydrofolate Dehydrogenase/Cyclohydrolase, Mitochondrial
}

National Cancer Institute

\section{Source}

National Cancer Institute. Bifunctional Methylenetetrahydrofolate

Dehydrogenase/Cyclohydrolase, Mitochondrial. NCI Thesaurus. Code C104945.

Bifunctional methylenetetrahydrofolate dehydrogenase/cyclohydrolase, mitochondrial (350 aa, $\sim 38 \mathrm{kDa}$ ) is encoded by the human MTHFD2 gene. This protein is involved in folate metabolism. 